\title{
Research and application of a new soil moisture sensor
}

\author{
Xingchao Zhang ${ }^{1, a}$, Chengzhi Yang ${ }^{1}$ and Lu Wang ${ }^{1}$ \\ ${ }^{1}$ Faculty of Electrical and Information Engineering, Oxbridge College, Kunming University of Science and Technology, Kunming \\ 650106, China
}

\begin{abstract}
The measurement of soil moisture is the basis for the refinement of agriculture to implement water-saving irrigation. A new soil moisture sensor was designed based on its theoretical basis and analysis of the dielectric constant characteristics of soil. It mainly consists of probes, electronic circuits and wiring cables. The calibration test of the sensor was conducted under the working voltage of $2.5 \sim 5.5 \mathrm{~V}$. Using the experimental data to analyze the regression function, it was found that the output voltage of the sensor should have a linear negative correlation with the volumetric moisture content of the soil, and the coefficient of determination $\mathrm{R}^{2}>0.986$. The accuracy of the sensor was verified by soil samples with different moisture content. The average absolute value of absolute errors is less than $2.5 \%$. The results show that the sensor has stable and reliable working performance, high measurement accuracy and good linearity, and is suitable for moisture measurement in most types of soil.
\end{abstract}

\section{Introduction}

Water is an important part of the soil and it is one of the most important factors affecting the growth and yield of crops ${ }^{[1]}$. The measurement of soil moisture is the basis for the refinement of agriculture to implement water-saving irrigation ${ }^{[2]}$. Accurate observation and calculation of soil moisture and establishment of a soil moisture content model have important practical implications for agricultural production ${ }^{[3]}$.

At present, the determination method of soil moisture could be classified into two categories. One is the change location sampling measurement. Such as drying and weighing method. The other is fixed position measurement. Such as neutron method, gamma ray method, time domain reflector method, frequency domain emitter method, sensor method. The drying and weighing method is simple in principle and high in accuracy, but it could not be continuously monitored in the field in real time. Neutron method and gamma ray method could monitor soil moisture content quickly and accurately, but there were problems of radioactive substances harmful to human health ${ }^{[4]}$. Considering the factors such as technical difficulty, cost, accuracy and real-time, the dielectric constant method based on the capacitance principle was more common. This method could realize the rapid and nondestructive measurement of soil moisture by using the indirect measurement method of soil dielectric property. The sensing components of the sensors used were mostly plug-in structures with simple geometric structures and easy fabrication. However, most of the measurement signals need conditioning, the circuit was relatively complex, and was limited by the size and strength of the sensing components, so it was not easy to measure the moisture content of deep soil ${ }^{[5]}$.

This paper mainly discussed a new type of soil moisture sensor with high performance and no damage. Its work performance is stable and reliable, with high measurement accuracy and good linearity. Research shows that the sensor can meet the requirements of practical application, and is suitable for most types of soil moisture measurement.

\section{Design principle of the sensor}

\subsection{Theoretical basis}

Modern physics believes that all nonmetals and metals under specific conditions belong to the dielectric. As a porous medium consisting of soil particles, water and air, the soil is also a special kind of dielectric. Its dielectric properties can be expressed, i.e.

$$
\varepsilon(\mathrm{f})=\varepsilon^{\prime}(\mathrm{f})-j\left[\varepsilon^{\prime \prime}(\mathrm{f})+\frac{\sigma}{2 \pi f \varepsilon_{0}}\right]
$$

Where $\varepsilon$ was soil's complex permittivity, $\varepsilon^{\prime}$ was dielectric constant, $\varepsilon^{\prime \prime}$ was dielectric loss, $\varepsilon_{0}$ was vacuum permittivity, $f$ was electromagnetic frequency, $\sigma$ was DC conductivity of soil.

According to the above formula, frequency is one of the main factors that affect the dielectric properties of soil. Selecting the appropriate electromagnetic frequency plays a key role in the measurement of soil moisture by using dielectric constant. When the electromagnetic

Corresponding author: a 854027031@qq.com 
frequency is $100 \mathrm{MHz}$, the dielectric constants of soil particles, water and air are about 4, 80 and 1 respectively. It can be seen that the moisture content of soil can directly affect its dielectric constant. Therefore, the soil moisture content can be measured by measuring the dielectric constant of the soil.

\subsection{The working principle of the sensor}

The $100 \mathrm{MHz}$ radio wave generated by the signal source was transmitted to the probe using a transmission line. Because the impedance of the transmission line was not matched with the impedance of the probe, some of the signals were reflected back in the transmission process to form a standing wave with the incident wave. This causes the amplitude of each point voltage on the transmission line to change. The dielectric constant of soil determines the probe impedance, so the change of voltage on the transmission line directly reflects the change of moisture in the soil. Its measurement equivalent circuit was shown in Figure. 1.

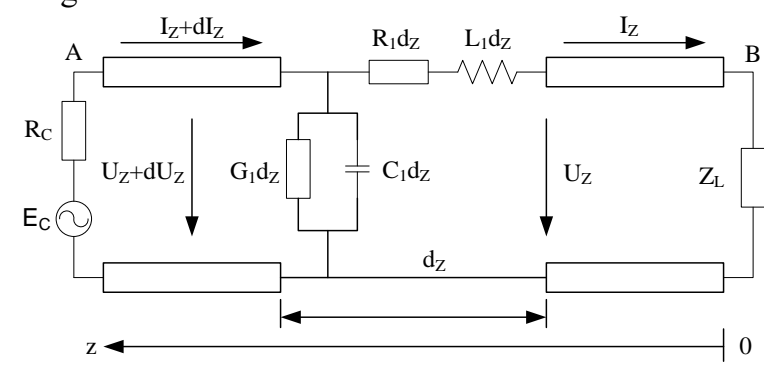

Figure 1. The measurement equivalent circuit

The transmission line used in this article was a coaxial cable, which was considered as a lossless transmission line. According to the theory of transmission line, the peak voltage of $\mathrm{A}$ and $\mathrm{B}$ can be obtained, i.e.

$$
\begin{aligned}
U_{A} & =\mathrm{A}(1+\rho) \\
U_{B} & =\mathrm{A}(1-\rho)
\end{aligned}
$$

When the length of the transmission line is equal to $1 / 4$ of the wavelength, the peaks and troughs of the standing wave are exactly at both ends of the coaxial cable. The voltage difference between $A$ and $B$ is:

$$
U_{A}-U_{B}=2 \mathrm{~A} \rho=2 \mathrm{~A} \frac{Z_{L}-Z_{C}}{Z_{L}+Z_{C}}
$$

Where the value of $A$ depends on the amplitude of the oscillator, and $Z_{\mathrm{L}}$ is the impedance of the probe, which is related to the dielectric constant of the soil. $Z_{C}$ is the characteristic impedance of the transmission line and $\rho$ is the reflection coefficient of the transmission line at point $\mathrm{A}$.

Due to the influence of soil dielectric constant, when the probe of the sensor was inserted into the soil, $\mathrm{Z}_{\mathrm{L}}$ changed with the change of soil moisture content, and then caused the change of the output voltage $\Delta U_{A B}$. Therefore, we can indirectly obtain soil moisture content by measuring the voltage difference at both ends of the transmission line.

\section{The structure of the sensor}

According to the working principle of the sensor, combined with its actual measurement requirements, the design of the structure of the soil moisture sensor was shown in Figure. 2.

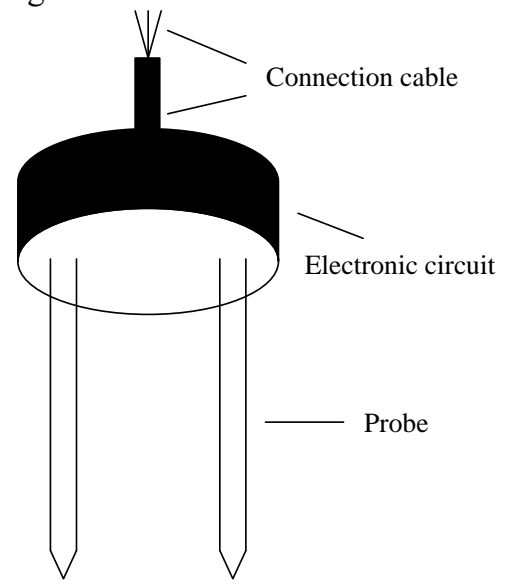

Figure.2. Appearance structure of soil moisture sensor

The probe electrode composed of a printed circuit board has two pin-type parallel structures and was electrically connected to the electronic circuit equipped with sensors, and was highly integrated to form an integrated structure. The probe ends were designed to be triangular in order to be easily inserted into the soil to be tested. After filling, the electronic circuit area was sealed with a rubber shell. Only a shielded three core wire was used as the external interface. The three core lines were respectively connected to the power input of the sensor, the ground wire and the signal output line.

\section{Test results and analysis}

\subsection{Calibration of the sensor}

When the temperature was $25^{\circ} \mathrm{C}$, the sensor was calibrated from 2.5 to $5.5 \mathrm{~V}$ supply voltage. The regression function and the coefficient of determination were shown in Table 1.

Table 1. Regression function of sensor output vs soil volumetric water content

\begin{tabular}{|c|c|c|}
\hline $\begin{array}{c}\text { Working } \\
\text { voltage } / \mathbf{V}\end{array}$ & $\begin{array}{c}\text { Regression } \\
\text { function }\end{array}$ & $\begin{array}{c}\text { Coefficient of } \\
\text { determination } \mathbf{R}^{\mathbf{2}}\end{array}$ \\
\hline 2.5 & $\mathrm{y}=-54.51 \mathrm{x}+91.92$ & 0.989 \\
\hline 3.0 & $\mathrm{y}=-48.41 \mathrm{x}+91.13$ & 0.990 \\
\hline 3.5 & $\mathrm{y}=-40.56 \mathrm{x}+89.73$ & 0.987 \\
\hline 4.0 & $\mathrm{y}=-35.58 \mathrm{x}+89.49$ & 0.986 \\
\hline 4.5 & $\mathrm{y}=-31.46 \mathrm{x}+89.15$ & 0.988 \\
\hline 5.0 & $\mathrm{y}=-28.13 \mathrm{x}+88.59$ & 0.988 \\
\hline 5.5 & $\mathrm{y}=-25.43 \mathrm{x}+88.19$ & 0.989 \\
\hline
\end{tabular}

The test results show that the output voltage of the sensor decreases with the increase of soil moisture content, and the output voltage of the sensor increases with the increase of operating voltage at a specific moisture content. Using the experimental data to analyze the regression function, it was found that the output voltage of the sensor should have a linear negative correlation with the volumetric moisture content of the 
soil, and the coefficient of determination $\mathrm{R}^{2}>0.986$.

\subsection{Verification of sensor}

In order to verify the measurement accuracy of the soil moisture sensor, the soil samples were tested with different volume moisture content. The test soil was air-dried, chopped and de-aggregated, and sieved through $1 \mathrm{~mm}$ and dried to constant weight. Then 20 parts of soil sample to be tested with different volumetric moisture content were mixed with water and sealed for $48 \mathrm{~h}$. The sensor was tested with a supply voltage of $3 \mathrm{~V}$. The test data was shown in Table 2. The results show that the average absolute value of the absolute error is less than $2.5 \%$, and the accuracy of the sensor is qualified.

Table 2. Results of testing and verification of sensor in $3 \mathrm{~V}$ supply voltage

\begin{tabular}{|c|c|c|}
\hline $\begin{array}{l}\text { Soil moisture } \\
\text { content } / \%\end{array}$ & $\begin{array}{c}\text { Measured } \\
\text { value } / \%\end{array}$ & Error $/ \%$ \\
\hline 2.5 & 1.16 & -1.34 \\
\hline 5.0 & 3.81 & -1.19 \\
\hline 7.5 & 8.16 & 0.66 \\
\hline 10.0 & 11.02 & 1.02 \\
\hline 12.5 & 14.86 & 2.36 \\
\hline 15.0 & 16.77 & 1.77 \\
\hline 17.5 & 18.72 & 1.22 \\
\hline 20.0 & 22.38 & 2.38 \\
\hline 22.5 & 21.85 & -0.65 \\
\hline 25.0 & 23.37 & -1.63 \\
\hline 27.5 & 25.74 & -1.76 \\
\hline 30.0 & 31.01 & 1.01 \\
\hline 32.5 & 32.11 & -0.39 \\
\hline 35.0 & 33.19 & -1.81 \\
\hline 37.5 & 37.86 & 0.36 \\
\hline 40.0 & 38.95 & -1.05 \\
\hline 42.5 & 41.74 & -0.76 \\
\hline 45.0 & 42.94 & -2.06 \\
\hline 47.5 & 48.08 & 0.58 \\
\hline 50.0 & 47.85 & -2.15 \\
\hline
\end{tabular}

\section{Conclusions}

This paper mainly discussed a new type of soil moisture sensor with high performance and no damage. The calibration test and analysis showed that the output voltage should have a linear negative correlation with the volumetric moisture content, and the coefficient of determination $\mathrm{R}^{2}>0.986$. It can be known from the accuracy measurement experiment that the average absolute value of absolute errors is less than $2.5 \%$. The results show that the sensor has stable and reliable performance, high accuracy and good linearity, and can meet the requirements of practical application, and it is suitable for water measurement of most types of soil.

\section{Acknowledgements}

This work was financially supported by Yunnan Provincial Education Department Scientific Research Foundation of China under Grant 2016ZZX306.

\section{References}

1. Li Lianjun, Sun Yurui, Lin Jianhui. Journal of Jiangsu University. 30, 541-544(2009). (In Chinese)

2. Luo Xiwen, Zang Ying, Zhou Zhiyan. Transactions of the CSAE. 22, 167-173(2006). (In Chinese)

3. Wang Xiaolei, $\mathrm{Hu}$ Jiandong, Jiang Min, et al. Transactions of the CSAE. 25,76-81(2009). (In Chinese)

4. Bai Zesheng. Control and Automation. 23, 202-203(2007). (In Chinese)

5. Li Jia'nian, Hong Tiansheng, Feng Ruijue, Yue Xuejun, Luo Yuqing. Transactions of the CSAE. 27, 216-221(2011). (In Chinese)

6. Shi Guoying, Zhang Bo. Agricultural equipment and vehicle engineering. 25-27(2009). (In Chinese) 